\title{
Improved Signal Detection Scheme for Co-Channel Interference Based on Fourth - order Cyclic Cumulants
}

\author{
Gaofeng Fana, Houde Quan and Hao Zhang ${ }^{b}$ \\ Department of Information Engineering, Ordnance Engineering College, Shijiazhuang 050003, \\ China; \\ afanr84@sina.cn, b18033828888@163.com
}

Keywords: Co-Channel Multi-Signal; Signal Detection; Cyclic Cumulants.

\begin{abstract}
Signal detection and the number of signal sources are the basis of dealing with the problem of co-channel multi-signal. An algorithm based on fourth-order cyclic cumulant is proposed for multisignal detection of co-channel and signal source number estimation. Firstly, the fourth-order cyclic cumulant of the received signal is calculated. The discrete spectral lines appear at the cyclic frequency, which is related to the signal carrier frequency. Then, the discrete spectral lines are extracted based on the morphological method. The number of discrete lines for signal detection and signal source number estimates. The algorithm does not need to set the threshold value, can carry on the signal detection and estimate the number of signal sources at the same time. For at most three arbitrary types of power mixed signals, such as BPSK, QPSK and MQAM, etc., in the signal to noise ratio of about $5 \mathrm{~dB}$ signal detection and signal source number of the correct rate is greater than $90 \%$, and the algorithm has good robustness to spectral overlapping degree.
\end{abstract}

\section{Introduction}

Signal detection and the number of signal source estimation is a more traditional problem in the field of communication signal processing, at present a large number of relevant research results have been published. Signal detection commonly used in energy detection method, which is susceptible to noise uncertainty, anti-noise performance is poor; eigenvalue decomposition detection method, requires multi-channel receiver, achieves more complex; cycle stationary detection method, this method usually need to set the threshold, flexibility is poor. The main problem is that the above algorithms are for single signal detection. The estimation of the number of signal sources is usually based on the array method. In this paper, the problem of multi-signal detection and signal source estimation in co-channel is studied in the single-channel receiver model. An algorithm based on fourth-order cyclic cumulant and discrete spectral line extraction is proposed. The complex stationary Gaussian white noise does not have high order cycle stationary characteristics, and its fourth-order cyclic cumulant is zero. For single-carrier signal carrier frequency and the number of signal source one-to-one correspondence, and the signal cycle frequency and carrier frequency, so according to the cyclic cumulant circulation frequency axis number of discrete spectral lines for the number of sources estimate. Noise is equivalent to the number of signal source is zero, can solve the signal detection and signal source number estimation problem. The algorithm does not need to set the threshold, which greatly improves the application flexibility.

The rest of this paper is organized as follows. Section II discusses the signal model of co-channel multi-signal. Section III introduces the cycle stationary properties analysis. The detection process is introduced in Section IV. The simulation of BPSK, QPSK and MQAM is given and the result is analyzed in Section V. Section VI gives conclusion of the paper.

\section{Cyclic Cumulant Algorithm}

The digital communication signal has cycle stationary after processing, such as forming, modulation and sampling. Cyclic cumulant is a commonly used cycle stationary analysis method. 
Theoretically, the high-order cyclic cumulants can completely suppress the stationary Gaussian noise, so that the signal can be analyzed well.

Suppose that $x(t)$ stands for zero mean cycle stationary signal, its n-order time-varying cumulant, $c_{x}(t ; \tau)_{n \cdot q}$ is a periodic function. Fourier series expansion coefficient, $c_{x}(\alpha ; \tau)_{n \cdot q}$ means n-order cyclic cumulants of $x(t)$ :

$$
\begin{aligned}
& c_{x}(\alpha ; \tau)_{n \cdot q}=\lim _{T \rightarrow \infty} \frac{1}{T} \int_{-T / 2}^{T / 2} c_{x}(t ; \tau)_{n \cdot q} \exp (-j 2 \pi \alpha t) d t \\
& c_{x}(t ; \tau)_{n \cdot q}=\operatorname{cum}\left\{x \exp (*)_{1}\left(t+\tau_{1}\right) \cdots x \exp (*)_{n}\left(t+\tau_{n}\right)\right\} \\
& =\sum_{\alpha \in A_{n \cdot q}^{o}} c_{x}(\alpha ; \tau)_{n \cdot q} \exp (j 2 \pi \alpha t)
\end{aligned}
$$

Where $(*)_{u}, u=1, \cdots, n$ denotes that the logarithm value may be conjugated, and the total number of conjugate operations is $q, \tau=\left\{\tau_{1}=0, \tau_{2}, \cdots, \tau_{n}\right\}$ is an n-dimensional delay vector, $A_{n \cdot q}^{\alpha}=\left\{\alpha: c_{x}(\alpha ; \tau)_{n \cdot q} \neq 0\right\}$ is cyclic frequency set of $c_{x}(\alpha ; 0)_{4 \cdot 0}$.

The fourth-order cyclic cumulant used in this paper $c_{x}(\alpha ; 0)_{4 \cdot 0}$ is:

$$
\begin{aligned}
& \left.\left.c_{x}(\alpha ; 0)_{4 \cdot 0}=\left\langle c_{x}(t ; 0)_{4 \cdot 0}\right\rangle_{t}=<x^{4}(t) \exp (-j 8 \pi \alpha t)\right\rangle_{t}-3<x^{2}(t) \exp (-j 4 \pi \alpha t)\right\rangle_{t}^{2} \\
& <\cdot>_{t} \text { Represents the time average. }
\end{aligned}
$$

The cycle frequency is:

$$
\alpha=k f_{b}+(n-2 q) f_{c}=k f_{b}+4 f_{c}, k=0, \pm 1, \pm 2, \cdots
$$

For the raised cosine signal, the majority of energy concentrated in the bandwidth, so usually the cycle frequency is:

$$
\alpha=4 f_{c} \pm k f_{b}, k=0, \pm 1
$$

It can be seen that the cyclic frequency of $\left|c_{x}(t ; 0)_{4.0}\right|$ corresponds to the carrier frequency and the code rate of the signal.

If the random variables $\left\{x_{i}\right\}$ and $\left\{y_{i}\right\}$ are statistically independent, the cumulate has a "semiinvariant", which is. :

$$
c\left(x_{1}+y_{1}, \cdots, x_{k}+y_{k}\right)=c\left(x_{1}, \cdots, x_{k}\right)+c\left(y_{1}, \cdots, y_{k}\right)
$$

Combined with the signal model of this paper:

$$
c_{x}(\alpha ; 0)_{4 \cdot 0}=\sum_{i=1}^{N} c_{s i}(\alpha ; 0)_{4 \cdot 0}
$$

So the cycle frequency of co-channel multi-signal is:

$$
\alpha=\bigcup_{i=1}^{N} \alpha_{i}=\bigcup_{i=1}^{N}\left(4 f_{c i}+k f_{b}\right), k=0,1
$$

The above two formulas show that the fourth-order cyclic cumulant of the mixed signal is equal to the sum of the fourth-order cyclic cumulants of the independent signals, and the cyclic frequency is the union of the signal cycle frequencies. This property fully demonstrates that the cyclic cumulant can reflect the cycle stationary characteristics of each independent signal and is suitable for dealing with the co-channel multi-signal problem.

Gaussian white noise is second-order stationary and does not have high-order cycle stationary characteristics, and its fourth-order cyclic cumulant is zero. The modulation signal has a high-order cycle stationarity, and its fourth-order cyclic cumulant is not zero at the cyclic frequency. Thus, $\left|c_{x}(\alpha ; 0)_{4.0}\right|$ can be used as the detection statistic to distinguish the signal from the noise.

In theory, the cyclic cumulant is not zero at the cyclic frequency $\alpha$, According to Equation 7 type of independent signal cycle frequency and carrier frequency one-to-one correspondence, so for singlecarrier signal when each individual signal carrier frequency is not equal, their cycle frequency is not equal. According to (10) type of common-channel signal cycle frequency for each independent signal 
cycle frequency union. Therefore, searching for the number of $\left|c_{x}(\alpha ; 0)_{4.0}\right|$ nonzero values on the cyclic frequency axis can provide an estimate of the number of independent signal sources in the co-channel signal.

\section{The Detection Process}

The cycle stationary properties reflected by the cyclic cumulants are of a progressive nature, that is, when the data tends to infinity. In practical applications, the cumulative amount of noise is generally not zero due to the limited length of the data. In particular, there is a maximum when $\alpha=0$, as shown in Figure 1.Likewise, the value of signal $\left|c_{x}(\alpha ; 0)_{4.0}\right|$ is not zero at the cyclic frequency, and truncation noise occurs. When the data is sufficiently long, the value at the cyclic frequency on the cyclic frequency axis is much larger than the value of the truncated noise, and a discrete spectrum appears as shown in Fig2. Therefore, according to the location of discrete lines can be achieved cochannel signal detection.

The value of $4 f_{c i}$ is better at noise resistance than the spectral line at symbol rate $4 f_{c i} \pm f_{b}$.

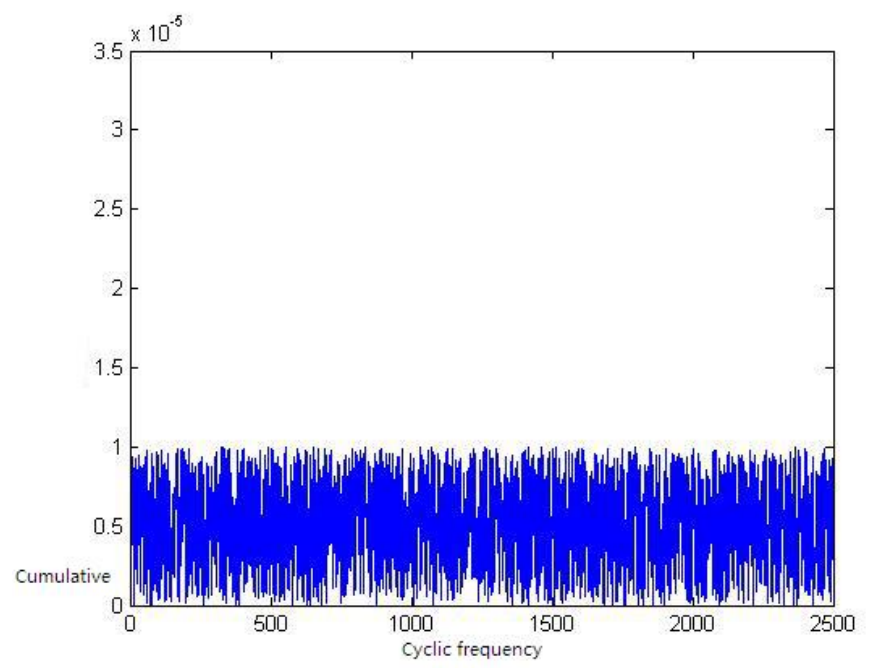

Fig. 1 Cumulative amount of noise

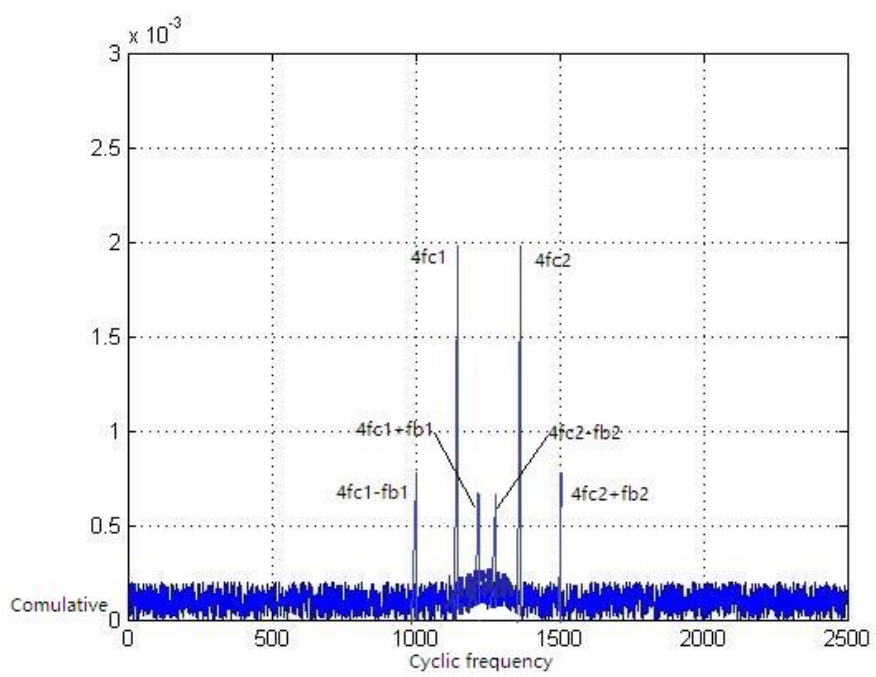

Fig. 2 Cumulative amount of Co-channel signals

According to the symmetry of the $4 f_{c i} \pm f_{b}$ line to remove the line combined with morphology based on the discrete spectral line extraction algorithm at 4 times the carrier frequency of the discrete lines can be extracted to achieve the number of signal sources of common channel estimation. 
It can be seen from Fig1, the actual value of the noise $\left|c_{x}(\alpha ; 0)_{4.0}\right|$ is not zero, and the signal detection is usually required to set the detection threshold. When the threshold is greater than the signal, otherwise there is no signal. The number of sources mentioned above is estimated based on the number of discrete lines on the frequency axis of the cycle, and it is often necessary to set the corresponding thresholds to use an effective number of discrete lines as an estimate of the number of sources. However, the specific value of $\left|c_{x}(\alpha ; 0)_{4.0}\right|$ and SNR and data length and so on, the threshold is not easy to set. This paper presents an algorithm for multi-signal detection and source estimation without co-channel threshold estimation.

Consider signal detection first. Given the finite data length, the maximum $\left|c_{x}(\alpha ; 0)_{4.0}\right|$ of the noise appears at $\alpha=0$, while for the IF signal there is a maximum at $\alpha=4 f_{c i}$ for $f_{s}>4 f_{c i}+f_{b i}$. Therefore, signal detection can be performed according to the difference between the $\left|c_{x}(\alpha ; 0)_{4.0}\right|$ maximum value positions.

Suppose that $H_{0}$ stands for no signal, $H_{1}$ stands for that there is a signal, the signal detection formula is expressed as:

$$
\left\{\begin{array}{l}
H_{0}, \arg \max _{\alpha}\left(\left|c_{x}(\alpha ; 0)_{4.0}\right|\right)=0 \\
H_{1}, \arg \max _{\alpha}\left(\left|c_{x}(\alpha ; 0)_{4 \cdot 0}\right|\right) \neq 0
\end{array}\right.
$$

Combined with signal detection, noise is equivalent to the number of signal sources is zero, the two can be unified.

\section{Simulation Analysis of the Proposed Method}

The performance of the algorithm is tested by AWGN channel. The independent signal types are BPSK, QPSK, and MQAM, with raised cosine shaping and a pulse shaping factor of 0.35 . Number of signal sources $\mathrm{N}=1,2,3$. Sampling rate $f_{s}=19200 \mathrm{~Hz}$, and data length 40960 samples.

Signal detection probability when the number of signal sources and signal to noise ratio changes.

The algorithm firstly removes the spectral lines related to the signal symbol rate when detecting and estimating the number of signal sources, so this is independent of the symbol rate. Suppose that the symbol rate of each signal is equal. The signal modulation parameter is:

$f_{c 1}=2400 \mathrm{~Hz}, f_{b 1}=1$ 200Baud, $f_{c 2}=2700 \mathrm{~Hz}, f_{b 2}=1200 \mathrm{Baud}, f_{c 3}=3300 \mathrm{~Hz}, f_{b 3}=$ 1200Baud.The signal detection probability is shown in Fig 3.

The probability of signal detection is greater than 0.9 when $\mathrm{SNR}=0 \mathrm{~dB}$, to 1

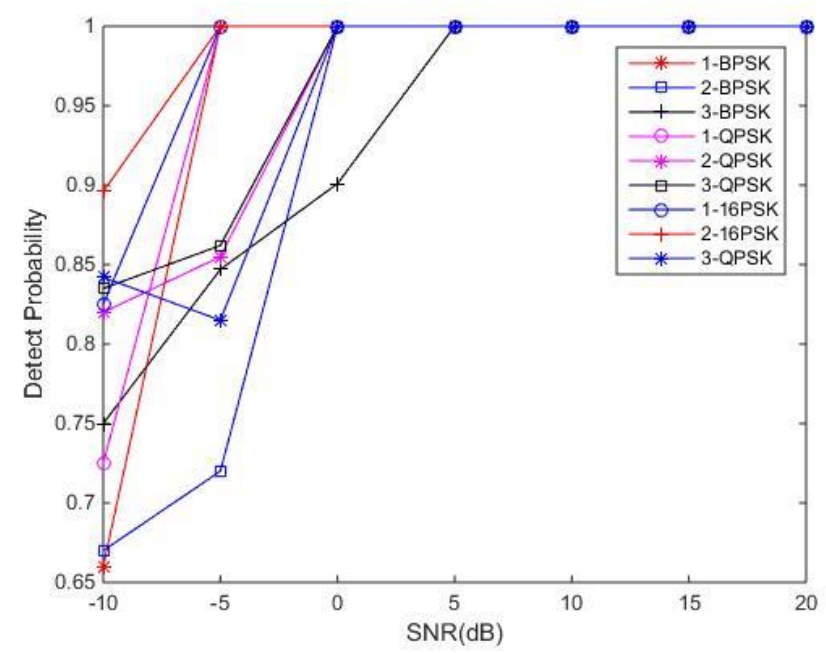

Fig.3 Probability of signal detection

when $\mathrm{SNR}=5 \mathrm{~dB}$. The algorithm has good anti-noise performance, which is mainly because of the noise and signal cycle stationary difference in the detection, coupled with the discrete spectral 
extraction algorithm also has some ability to suppress noise, the combination of the two makes the algorithm have a good inhibition of noise, and the detection probability is still high under low SNR.

\section{Summary}

In this paper, the fourth-order cyclic cumulant and morphological discrete spectral line extraction algorithm are used to detect the co-channel multi-signal and estimate the number of signal sources. The algorithm has good anti - noise performance, and the correctness of the signal detection probability and the number of signal source estimation are high at low signal - to - noise ratio, and the algorithm has good robustness to the spectral overlapping degree of each independent signal. Compared with the traditional algorithm, the biggest advantage is that the number of detection and signal source estimation is united, and there is no need to set the threshold in advance, and the practical application has very good flexibility. But also according to the needs of the signal spectrum of the pre-estimated, so that can greatly reduce the calculation of the algorithm.

\section{References}

[1]. Urkowitz H. Energy detection of unknown deterministic signals [J].Proceedings of the IEEE, 1967, 55(4):523-531.

[2]. Wax M, Kailath T. Detection of signals by information theoretic criteria [J]. IEEE Transactions on Acoustics, Speech and Signal Processing, 1985, 33(2): 387-392.

[3]. Gardner W A, Spooner C M. The Cumulant Theory of Cycle stationary Time-Series, Part I: Foundation [J]. IEEE Transactions on communications, 1994, 42(12): 3387-3406.

[4]. Jin Yan, Ji Hongbing, Luo Junhui. A Cyclic-Cumulant Based Method for DS-SS Signal Detection and Parameter Estimation [J]. Chinese Journal of electronics, 2006, 04(4): 634-637.

[5]. Huang Wenfang, GONG Shan, JIANG Hua. Improved Spectrum Sensing Algorithm Based on Second-Order Cyclic Cumulants [J].Journal of Information Engineering University, 2011,12 (5): 574-577. 